\title{
O peracionalizando as categorias acesso e descentralização na análise de sistemas de saúde
}

\author{
O perationalizing the categories access \\ and decentralization in health systems analysis
}

Virginia Al onso Hortale 1

Manoela Pedroza 1

Maria Luiza Garcia Rosa 2

\footnotetext{
1 Departamento de Administração e Planejamento em Saúde, Escola Nacional de Saúde Pública, Fundação Oswaldo Cruz. Rua Leopoldo Bulhões 1480, 7o andar, Manguinhos, Rio de Janeiro, RJ 21045-900, Brasil. virginia@ensp.fiocruz.br 2 Consórcio Brasileiro de Acreditação - CBA. Rua São Francisco Xavier 524, bloco E, 2o andar, Maracanã, Rio de Janeiro, RJ, Brasil.
}

Abstract This paper presents a proposal for operationalization of access and decentralization in a health systems analytical model. It supports the hypothesis that decentralization of health systems fosters better access by users. This proposal is intended to contribute to a closer link between these categories and observed reality.

Key words Health Services Accessi bility; Decentralization; Health Systems

Resumo O artigo apresenta proposta de operacional ização das categorias acesso e descentrali zação em modelo de análi se de sistemas de saúde. Admi te a hi pótese de que se o sistema de saúde é descentralizado, el e vai permitir mai or acesso dos usuári os ao sistema. Pretende-se com essa proposta contribuir com uma maior vinculação dessas categorias na real i dade observada.

Palavras-chave Acesso aos Serviços de Saúde; Descentralização; Si stemas de Saúde 
Introdução

O objetivo deste artigo é apresentar proposta de operacionalização das categorias acesso e descentralização em modelo de análise de sistemas de saúde. É o terceiro de uma série de três artigos que são produto de discussões provenientes de estudo que se voltou para a construção e operacionalização de um modelo de análise de sistemas de saúde em uma perspectiva comparada.

No primeiro artigo da série (Hortale et al., 1999), apresentamos os diferentes modelos propostos para descrever os determinantes de saúde, os serviços de saúde ou o sistema de saúde em geral e justificamos a possibilidade de utilizar as categorias acesso e descentralização como possíveis categorias na construção do modelo. Em um segundo artigo, ainda inédito, aprofundamos a discussão dessas categorias, argumentando a favor da associação entre elas, por: sua capacidade de serem úteis à tomada de decisões; seus valores variarem em função do objeto que eles querem medir; permitirem comparações temporais; apresentarem uma certa variabilidade no tempo e no espaço; e terem um caráter de exclusividade mútua.

Nosso pressuposto, já explicitado nos artigos anteriores, é que a saúde do indivíduo é responsabilidade social. Para tanto, na operacionalização do modelo, consideramos duas dimensões: a social e a política.

Na dimensão social, o acesso é considerado como a categoria fundamental, independente das justificativas que possam ser dadas para a sua não-efetivação (custos excessivos, habilidade técnica, características da organização, etc.). O acesso deve funcionar no modelo como uma categoria-valor, ou seja, uma referência a ser atingida em qualquer serviço e ser o orientador de todas as políticas. Sua avaliação não pode se dar de forma simples, portanto não podemos perder de vista que ele também deve abranger os vários planos do processo de produção de serviços.

$\mathrm{Na}$ dimensão política, a descentralização não é considerada um val or e um fim em si mesma, mas uma condição necessária para melhorar o acesso, a adequação da resposta social, a participação, a qualidade, a sustentação e a eqüidade no campo da saúde. Consideramos que um sistema de saúde descentralizado, em uma dinâmica de transferência de recursos e autoridade às diferentes instâncias do sistema de saúde, causa um impacto positivo na gestão e nas diferentes modalidades de atenção e dá oportunidade para que os processos de refor- ma permitam a geração e desenho de novos modelos de atenção, papéis e funções, modalidades de capacitação, sistemas de remuneração e novas formas de participação das instituições, sindicatos e setores acadêmicos. Sua análise deve levar em consideração a missão e as funções do Estado, os serviços de saúde e os usuários do sistema no marco das características históricas e institucionais inerentes a cada realidade nacional.

Pretendemos que o modelo seja avaliatório e complexo, englobando funções sociais, culturais, racionais e produtivas em diversos planos e condicionantes externos. Na sua construção numa perspectiva comparada, levamos em consideração os limites impostos pelo estado da arte, pois, nas áreas de avaliação de políticas e de sistemas de saúde, as análises ainda carecem de maior profundidade pela escassez metodológica dos instrumentos utilizados, mesmo tendo alguns autores procurado fazer aproximações através de categorias ainda gerais.

No Brasil, em particular, essa abordagem, apesar de ter sido introduzida há al guns anos nas instituições de ensino e pesquisa da área de saúde pública, o foi de forma fragmentada e pouco sistemática. Fomos buscar um maior rigor metodológico nas ciências políticas, já que, nessa área, a abordagem comparada se caracteriza por ser uma ferramenta antiga, bem desenvolvida e um instrumento metodológico precioso (Heidenheimer, 1986; Mény, 1993).

Baseamo-nos em três principais autores, de quem retiramos os elementos que nos ajudam na construção do modelo e operacionalização das categorias acesso e descentralização, admitindo a hipótese de que, se o sistema de saúde é descentralizado, ele vai permitir maior acesso dos usuários ao sistema.

Contandriopoulos (1986, 1990, 1994), autor que vem contribuindo na discussão da abordagem comparada de sistemas de saúde, propõe um modelo ampliado de sistema de saúde, no qual interagem dois circuitos: o do estado de saúde e o de cuidados. Este modelo possibilita o estabel ecimento de relações entre os diversos componentes e introduz al gumas possibilidades de avaliação de sistemas de saúde complexos como são os existentes. Também se baseia na idéia de que o sistema de saúde está em crise e que esta pode ser caracterizada em quatro dimensões: financiamento, regulação, valores e conhecimento. Sugere al gumas categorias de análise localizadas nestas dimensões, dentre elas as de acesso e de descentralização.

Parsons (1951, 1956, 1977, apud Sicotte et al., 1996) introduz a discussão do sistema social e, por conseqüência, das características 
bási cas do sistema de saúde. Finalmente, Sicotte et al. (1996) contribuem com a idéia da viabilidade de construção de um modelo complexo e multidimensional, quando apresentam um modelo de desempenho das organizações, cujo plano de análise nos ajuda a entender as peculiaridades do sistema de saúde enquanto um conjunto de organizações ou subsistemas.

\section{O modelo, seus pressupostos}

e suas dimensões de análise

Os modelos analíticos de sistemas de saúde mais conhecidos utilizam as seguintes categorias: níveis de gerência (administração, planejamento, etc.); produção de recursos (medicamento, conhecimentos); organizações de recursos (públicas, privadas, ONGs); financiamento e prestação de serviços (Roemer, 1991). No entanto, eles não se adaptam à nossa idéia de modelo de análise, porque têm uma preocupação excessiva com a estrutura interna, deixando em segundo plano as relações com o meio, os interesses dos atores envolvidos, as dinâmicas complexas da população.

Contandriopoulos (1986:7) considera que a análise deve levar em conta o meio ambiente ou o contexto cultural, social, político, econômico, tecnológico, além dos valores culturais e ideológicos da sociedade em estudo. Para ele, "a análise da dinâmica do si stema de saúde e das características específicas dos serviços de saúde vão nos levar a defi nir as características que são comuns a todos os si stemas de saúde, ou seja, as 'rigidezes incontornáveis' que constituem o esquel eto da organização de todos os sistemas de saúde, assim como os elementos que levam ao crescimento desses si stemas e os que permitem controlá-lo".

Para ele, os elementos constitutivos do sistema de saúde são quatro: os recursos, os serviços, o estado de saúde e os fatores determinantes dos problemas de saúde. Sua compreensão do funcionamento do sistema se faz a partir de três fatores:

- identificação dos principais grupos de agentes que intervêm nesse sistema;

- as relações que existem entre eles;

- apreciação das principais motivações desses agentes.

A grande contribuição desse autor na concepção do modelo se dá na discussão das particularidades dos serviços de saúde, sendo a mais importante delas a que diz respeito à intervenção do poder público ser indispensável (regulação), em virtude das características in- trínsecas dos serviços de saúde, que são a incerteza ligada à ocorrência da doença e ao estado de saúde futuro de cada indivíduo, ao desequilíbrio da informação que existe entre o indivíduo doente e o profissional (por causa da indeterminação que existe sobre os efeitos dos serviços médicos) e a utilização dos serviços para satisfazer necessidades individuais através de outras pessoas que não o indivíduo ("externalidade"). Nesse caso, a noção abrangente de "externalidade" tem conseqüências importantes na elaboração de políticas de saúde, ou seja, "quanto mais abrangente for a noção de externali dade, as respostas no plano da necessária regulação serão mais i mportantes" (Contandriopoulos, 1986:19).

No que diz respeito às nossas categorias de análise, a contribuição deste autor é essencial, pois nos estimula a ser criativos, quando apresenta suas idéias de uma utopia na reforma dos sistemas de saúde (Contandriopoulos, 1994), que, em resumo, são: manter a universalidade da cobertura e o acesso dos serviços, graças à um financiamento público do sistema; e descentralizar as decisões para a organização e o funcionamento do sistema de saúde.

Sicotte et al. (1996), ao desenvolverem uma proposta conceitual integradora para a análise do desempenho de serviços de saúde, visando tanto superar a fragmentação existente na variedade de model os de desempenho organizacionais quanto incorporar o caráter multidimensional e facilmente paradoxal do desempenho do serviço, se aproximam da nossa idéia de integração e multidimensionalidade do sistema de saúde.

Esses autores baseiam-se em Henry Mintzberg (Mintzberg, 1982, apud Sicotte et al., 1996), autor que desenvolveu estudos sobre a estrutura e dinâmica das organizações em geral. Este autor considera os serviços de saúde como burocracias profissionais que apresentam duas especificidades principais. A primeira é a de ser um serviço de natureza pública. Embora seu papel social seja preservar e promover a saúde da população e dos indivíduos e seja objeto de um grande consenso social, a definição e a medição de resultados ainda são complicadas.

A segunda é a de que o meio ambiente político, legal, financeiro que confronta as organizações de saúde é extremamente complexo e pluralista, requerendo o desenvolvimento e manutenção de ligações intra e intersistêmicas. Ao visualizarmos a construção do modelo, admitimos a possibilidade de que ele funcione de forma ampliada em relação às especificidades descritas acima. 
A grande contribuição desses autores está na aceitação e posterior adaptação do modelo parsoniano de sistema social, considerando que esse modelo permite explicitar os vários mecanismos que são importantes no monitoramento do desempenho da organização. Como nossa perspectiva é sistêmica, vislumbramos uma possibilidade de, ao adotar essa abordagem, estarmos avançando na construção do modelo.

O modelo de Parsons de sistema social (Parsons, 1951, apud Sicotte et al., 1996) apresenta quatro principais funções - ou exigências - que todo sistema social (e podemos dizer, o sistema de saúde) deve cumprir para sobreviver: ser orientado por objetivos; interagir com seu meio ambiente para adquirir os recursos necessários para a sua transformação; integrar seu processo interno para garantir a produção e manter os valores e normas que ajudam e obrigam as três. Nessa perspectiva, o conceito de equilíbrio é central.

Para Sicotte et al. (1996), o que torna o modelo parsoniano interessante na construção do modelo deles é a sua capacidade de:

a) englobar os vários modelos dominantes de desempenho organizacional, respectivamente o modelo de objetivos racionais, o modelo de aquisição de recursos, o modelo de processo interno de decisão e o modelo de relações humanas;

b) apresentar uma forte estrutura integradora, na qual a complementaridade do desempenho das várias organizações de saúde está bem integrada enquanto a especificidade de cada uma dessas perspectivas está bem preservada;

\section{Figura 1}

Subsistemas funcionais dos serviços de saúde e seus subsistemas inter-relacionados.
Meios

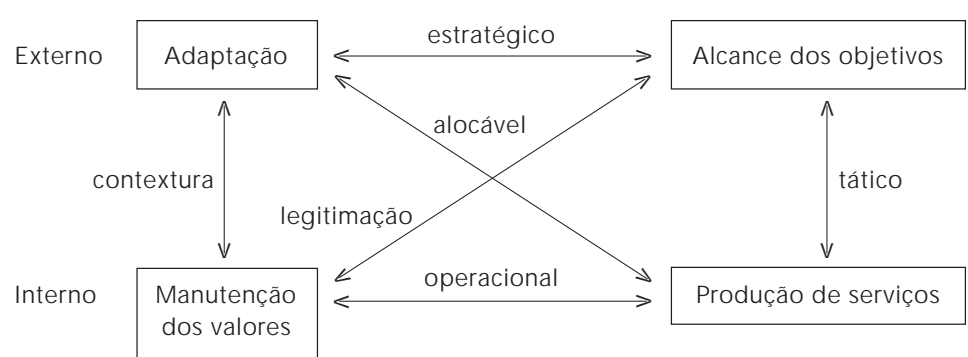

Fonte: extraído de Sicotte et al., 1996. c) enriquecer o conceito de desempenho da organização de saúde, tornando visíveis várias dimensões do desempenho que são freqüentemente negligenciadas.

Embora nossa perspectiva não seja a avaliação de desempenho do sistema de saúde, admitimos a possibilidade de aproveitar os principais elementos do modelo parsoniano na construção do modelo, os quais discutiremos a seguir.

O modelo parsoniano considera que são quatro as funções que se estruturam na interrelação entre dois eixos: vertical e horizontal (Figura 1). O eixo vertical (interno-externo), descreve a relação entre o sistema aberto e seu meio ambiente, e o eixo horizontal (meiosfins), o equilíbrio entre a entrada de recursos (material e simbólico), seu processamento até o ponto de ser usado, seu consumo real e os resultados obtidos. Os quatro subsistemas funcionais estão posicionados de acordo com os dois eixos acima.

A função adaptação (externo/meios) assegura o crescimento e a sobrevivência institucional através do manuseio criterioso das oportunidades e ameaças presentes no ambiente. A função alcance dos objetivos (externo/ fins) capacita a organização a fazer escolhas estratégicas que resultem no alcance de objetivos de longo prazo. A função produção de serviços (interno/fins) é o centro da organização (incluindo serviços clínicos e de suporte). A função manutenção dos valores produz razão, compreensão e coesão entre os membros da organização. Essa função gera o sistema de valores em que repousam as outras três funções.

Na medida em que essas funções não são independentes umas das outras, mas reciprocamente interligadas, Sicotte et al. (1996:18), baseando-se em Parsons, definem seis formas de intercâmbio que asseguram a coesão e o equilíbrio do sistema (Figura 1): estratégica (adaptável $\leftrightarrow$ alcance dos objetivos); alocável (adaptação $\leftrightarrow$ produção de serviços); tática (alcance dos objetivos $\leftrightarrow$ produção de serviços); operacional (manutenção de valores $\leftrightarrow$ produção de serviços); legitimação (manutenção de valores $\leftrightarrow$ alcance dos resultados); e contextura (adaptação $\leftrightarrow$ manutenção de valores).

Procuramos considerar, na construção do modelo, um valor pelo qual possamos avaliar o sistema, pois toda avaliação pressupõe um ideal a ser atingido. Sicotte et al. (1996) observaram que os tradicionais model os de avaliação dos serviços refletem diferentes concepções de meios, fins, gerências e estruturas (ou seja, diferentes modelos organizacionais), não podendo haver um modelo universal. Uma característica universal é aquela que pode dar 
conta de otimizar, ao mesmo tempo, todas as funções que acabamos de citar, demandas e objetivos de uma organização, de maneira unívoca e homogênea.

Partimos, também, da concepção de que a articulação de um sistema complexo se faz em nove níveis, descritos por Le Moigne (1990):

1) Fenômeno é identificável, o que o diferencia de seu meio ambiente.

2) Fenômeno é ativo, isto é, ele faz.

3) Fenômeno é regulado, pois não sabemos modelar o caos.

4) Fenômeno informa-se sobre seu próprio comportamento, isto é, tudo se passa como se o sistema produzisse, de maneira endógena, formas intermediárias, informações, sistemas de símbolos, que asseguram a intermediação com a regulação.

5) Sistema decide o seu comportamento, ou seja, é capaz de gerar informação e suas próprias decisões.

6) Sistema memoriza, pois ao elaborar suas decisões não considera automaticamente as informações que recebe. Os dois níveis anteriores ( 4 e 5), juntamente com este, formam o arquétipo operação-informação-decisão e serão apresentados mais adiante como as principais dimensões na operacionalização do modelo proposto.

7) Sistema coordena suas decisões de ação.

8) Sistema imagina e concebe novas decisões possíveis, ou seja, é capaz de elaborar novas formas de ação.

9) Sistema é capaz de decidir sobre sua decisão, isto é, de se finalizar.

$\mathrm{O}$ arquétipo decisão-operação-informação pode ser caracterizado como três subsistemas. O nível das decisões permite à organização, em função das informações que recebe, comparar resultados, compreender as diferenças, conceber ações alternativas e escolher planos de ação pertinentes. O nível das operações é aquele onde o sistema age sobre os inputs e os transforma. O nível das informações permite à organização recolher informações sobre suas operações, sobre o meio ambiente e sobre ela mesma (Rodrigue, 1990).

A nossa proposta de modelo é feita com base em concepções anteriores, buscando operacionalizá-las a partir de alguns indicadores de avaliação.

Os fatores e objetivos gerais apresentados serão detalhados de forma específica e distinta em cada instância institucional (central ou local) e perante a população usuária. Procuramos não perder de vista a noção do valor norteador do acesso para cada subsistema de cada instância e a noção da descentralização como uma condição necessária para melhorar o acesso. É aqui que reside a complexidade de modelar e avaliar esta dinâmica. A seguir, apresentamos nossa proposta de construção teórica destas duas categorias.

\section{Acesso}

Procurando pensar o que seria a organização orientada pelo valor acesso, suas instâncias podem ser vistas da seguinte maneira:

1) Instâncias central elocal: é onde se promove e se concretiza a responsabilidade com as necessidades da população e com os valores sociais. É onde se avalia a efetividade na produção de resultados na saúde da população. É onde se dá a devida relevância das escolhas estratégicas com os processos de adaptação (objetivos pertinentes aos recursos, às necessidades da população e ao mercado). É onde toma forma a coerência dos serviços com as necessidades da população. E, finalmente, é onde se dá a adequação das escolhas de objetivos à demanda dos cuidados.

2) População/usuários: é onde se manifesta a legitimidade e confiança no sistema de saúde. É onde são revelados os fatores sócio-culturais que predispõem ou não o uso do sistema de saúde formal e as atitudes que influenciam o comportamento individual, como as crenças sobre os médicos e os serviços médicos, orientações pessoais sobre a saúde.

Dada essa caracterização, a categoria acesso é operacionalizada considerando os três principais subsistemas: de decisão, de operação e de informação.

\section{Subsistema de decisão}

É onde se dá a comparação dos resultados das operações com o projeto do sistema, a compreensão dos desvios, a concepção de alternativas e as escolhas de planos de ação. É o nível estratégico, tático e alocável de uma organização. Neste subsistema, o valor acesso está relacionado ao controle social, entendido como participação real da população na administração dos serviços, associada à gestão técnica e interna (indo contra a tendência crescente de profissionalização e burocracia).

O objetivo a ser atingi do por esse controle e essa participação é o de otimizar a função de interação com o meio (adaptável), e a avaliação se dá pela existência e condição de operação de mecanismos concretos de participação popular: conteúdo do processo, mecanismos de participação, finalidade das experiências. 
Segundo Forest (s/ d:144), "achar que toda ação pública deveria ser concebida como um processo aberto, conduzido de maneira a informar, escutar e a levar em conta os cidadãos não é puramente uma orientação ideológi ca, mas uma questão de efi cácia. O consenti mento e a iniciati va dos ci dadãos são condições necessárias ao al cance dos obj eti vos governamentais e, por isso, devem ter sua partena definição e execução das prioridades públicas".

A operacionalização a partir dos níveis do sistema de saúde pode ser vista na Tabela 1.

Subsistema de operação/produção de serviços

É o nível no qual o sistema age sobre a forma inicial de um conjunto de fatores (paciente, patologia, estrutura física da própria organização) e os transforma, no tempo, em uma forma distinta. É onde se dá, também, o reconhecimento mútuo (encontro do paciente, do terapeuta e da estrutura do serviço); a identificação da demanda, dos serviços e do projeto; a avaliação do problema de saúde (diagnóstico, investigação, especialização das estruturas de tratamento); as intervenções para regular o problema; e a continuidade das funções anteriores.

O valor acesso neste subsistema avalia a eqüidade (universalidade, gl obalidade e acessibilidade regional, social e organizacional); a igualdade nos serviços calcada em prioridades de áreas, problemas e grupos de população; a qualidade (continuidade, qualidade técnica, pertinência e satisfação); e a eficácia (rendimento, disponibilidade) dos serviços. A operacionalização a partir dos níveis institucionais pode ser vista na Tabela 1.

O peracionalização do acesso.

\begin{tabular}{|c|c|c|c|}
\hline $\begin{array}{l}\text { Dimensões } \\
\text { do conceito }\end{array}$ & Instância central & $\begin{array}{l}\text { bníveis do sistema } \\
\text { Instância local }\end{array}$ & População \\
\hline $\begin{array}{l}\text { Objetivos } \\
\text { e valores }\end{array}$ & $\begin{array}{l}\text { - responsabilidade } \\
\text { com as necessidades } \\
\text { da população } \\
\text { - relevância das escolhas } \\
\text { estratégicas } \\
\text { - coerência dos serviços } \\
\text { - adequação das escolhas } \\
\text { de objetivos }\end{array}$ & $\begin{array}{l}\text { - qualidade (continuidade, } \\
\text { globalidade, pertinência } \\
\text { e satisfação) } \\
\text { - eqüidade (universali- } \\
\text { dade, acessibilidade } \\
\text { regional, social } \\
\text { e organizacional) }\end{array}$ & $\begin{array}{l}\text { - produção de resultados } \\
\text { - ensino/pesquisa/ } \\
\text { extensão } \\
\text { - legitimidade e confiança } \\
\text { no serviço de saúde } \\
\text { (fatores predisponentes) }\end{array}$ \\
\hline $\begin{array}{l}\text { Subsistema } \\
\text { de decisão }\end{array}$ & $\begin{array}{l}\text { - estratégias (tipos } \\
\text { de gestão, regulação } \\
\text { e planejamento), } \\
\text { - táticas, (nível financeiro) } \\
\text { - alocação de recursos } \\
\text { e de autoridade }\end{array}$ & $\begin{array}{l}\text { - adaptação da } \\
\text { dimensão geográfica } \\
\text { - autonomia } \\
\text { (liberdade de escolha } \\
\text { e autofinanciamento) }\end{array}$ & $\begin{array}{l}\text { - mecanismos de } \\
\text { participação popular } \\
\text { (assembléias locais) } \\
\text { - controle social } \\
\text { (responsabilidade } \\
\text { com o sistema) }\end{array}$ \\
\hline $\begin{array}{l}\text { Subsistema de } \\
\text { operação/produção } \\
\text { de serviços }\end{array}$ & $\begin{array}{l}\text { - fatores de } \\
\text { acessibilidade } \\
\text { organizacional }\end{array}$ & $\begin{array}{l}\text { - coerência dos serviços } \\
\text { com as necessidade } \\
\text { da população, recursos } \\
\text { e objetivos } \\
\text { - acessibilidade } \\
\text { financeira, geográfica } \\
\text { e organizacional }\end{array}$ & $\begin{array}{l}\text { - uso dos serviços } \\
\text { (fatores capacitantes*) }\end{array}$ \\
\hline $\begin{array}{l}\text { Subsistema de } \\
\text { informação e } \\
\text { retroalimentação }\end{array}$ & $\begin{array}{l}\text { - processamento das } \\
\text { informações recebidas } \\
\text { - fomento de políticas } \\
\text { educacionais e } \\
\text { informativas } \\
\text { - mecanismos de } \\
\text { difusão/comunicação } \\
\text { eficientes }\end{array}$ & $\begin{array}{l}\text { - prestação de contas } \\
\text { para centro e população } \\
\text { - liberdade de pagamento }\end{array}$ & $\begin{array}{l}\text { - liberdade de escolha } \\
\text { - informações sobre } \\
\text { o tratamento } \\
\text { - alteração de fatores } \\
\text { predisponentes }\end{array}$ \\
\hline
\end{tabular}

* Fatores capacitantes: fatores que possibilitam a necessidade percebida se tornar uma demanda real de serviços. Representam custos para o prosseguimento dos cuidados: de conveniência, oportunidade, financeiros, físicos, de transporte, de esforço, de perdas de trabalho e repouso, etc. A expectativa é de que o aumento do peso destes fatores aumente a chance do indivíduo interromper os cuidados médicos. 
Subsistema de informação

\section{e retroalimentação}

É o que permite à organização recolher informações sobre suas operações, sobre o meio e sobre ela mesma. Visa a receber, selecionar e tratar as informações recebidas; guardar o traço dos implexos anteriores; passar informações para os subsistemas de operação e de decisão.

O acesso nessa dimensão é avaliado pela ação retroativa de todo processo: prestação de contas, democratização de resultados de ensino-pesquisa-serviços, realização de atividades educacionais, integração do sistema com outros e entre os seus subníveis, etc.

O que se avalia é a possibilidade de os usuários terem autonomia de decisão, clareza no tratamento, opções pessoais e a influência dos fatores predisponentes, pela ampliação da percepção social da utilidade e do valor das organizações de serviços médicos. Os fatores predisponentes seriam os fatores sócio-culturais que existem previamente à doença e que propiciam ou não o uso do sistema de saúde formal; atitudes que influenciam o comportamento individual, como as crenças sobre os médicos e os serviços médicos; orientações pessoais sobre a saúde e outras dimensões psicossociais. A operacionalização a partir dos níveis institucionais pode ser vista na Tabela 1.

\section{Descentralização}

Dentro da mesma lógica utilizada na operacionalização do valor acesso, sistematizamos as instâncias da descentralização da seguinte maneira:

1) Instância central e local: é onde se define o grau de descentralização dos recursos, da autoridade e das competências. É onde fica caracterizada a autonomia dos serviços e a desconcentração de competências.

2) População/ usuários: é onde são incorporados os setores excluídos ou marginalizados.

A categoria descentralização também é operacionalizada, considerando-se os três principais subsistemas: de decisão, de operação e de informação.

\section{Subsistema de decisão}

Considerando os princípios de autonomia do serviço, desconcentração de competências e participação nas decisões de interesse geral, essa categoria é aval iada concretamente pela responsabilidade com os objetivos centrais e necessidades locais, assim como pela desconcentração das decisões estratégicas. É também avaliada pela sua capacidade de adquirir recursos, de decidir de forma autônoma e pelo grau de desconcentração das decisões tático-operacionais. Para a população, sua participação é avaliada nas decisões de interesse geral. A operacionalização a partir dos níveis do sistema de saúde pode ser vista na Tabela 2.

\section{Subsistema de operação/produção de serviços}

Considerando o princípio da desconcentração de competências, são avaliadas a coerência com o planejamento central, sua integração com outras partes do sistema, a prestação de contas e, para a população, a criação de instâncias representativas e acesso à informação. A operacionalização a partir dos níveis institucionais pode ser vista na Tabela 2.

\section{Subsistema de informação e retroalimentação}

Considerando o princípio da participação nas decisões de interesse geral, são avaliados os critérios e indicadores estratégicos e táticos de acompanhamento da gestão. Para a população, é avaliado o acompanhamento da gestão. A operacionalização a partir dos níveis institucionais pode ser vista na Tabela 2.

\section{Considerações finais}

Este artigo apresentou a proposta de operacionalização das categorias acesso e descentralização em modelo de análise de sistemas de saúde. Para tanto, justificou, com base em alguns autores, a forma e a operacionalização dessas categorias, tendo em vista que o modelo se propôs avaliatório e complexo, englobando funções sociais, culturais, racionais e produtivas em diversos planos e condicionantes externos.

O acesso funciona no modelo proposto como uma categoria-valor, e sua avaliação não pode se dar de forma simples, devendo abranger os vários planos do processo de produção de serviços. Como pode ser percebido, as dimensões que compõem a categoria abarcam fatores bem distintos da mera acessibilidade aos serviços, sendo esta apenas um deles. Como é possível ver tudo isso em um mesmo conjunto chamado acesso? Para isso, é preciso mudar o referencial de construção desse conceito, normalmente utilizado e anteriormente exposto. 
Tabela 2

O peracionalização da descentralização.

\begin{tabular}{|c|c|c|c|}
\hline $\begin{array}{l}\text { Dimensões } \\
\text { do conceito }\end{array}$ & Instância central & $\begin{array}{l}\text { níveis do sistema } \\
\text { Instância local }\end{array}$ & População \\
\hline $\begin{array}{l}\text { Objetivos } \\
\text { e valores }\end{array}$ & $\begin{array}{l}\text { - grau de descentra- } \\
\text { lização de recursos } \\
\text { - transferência de } \\
\text { autoridade, competências } \\
\text { e serviços }\end{array}$ & $\begin{array}{l}\text { - autonomia do serviço } \\
\text { - desconcentração } \\
\text { de competências }\end{array}$ & $\begin{array}{l}\text { - incorporação dos } \\
\text { setores excluídos } \\
\text { ou marginalizados }\end{array}$ \\
\hline $\begin{array}{l}\text { Subsistema } \\
\text { de decisão }\end{array}$ & $\begin{array}{l}\text { - responsabilidade } \\
\text { com objetivos centrais } \\
\text { e necessidades locais } \\
\text { - desconcentração das } \\
\text { decisões estratégicas }\end{array}$ & $\begin{array}{l}\text { - capacidade de adquirir } \\
\text { recursos } \\
\text { - capacidade de decidir } \\
\text { de forma autônoma } \\
\text { - desconcentração das } \\
\text { decisões tático- } \\
\text { operacionais }\end{array}$ & $\begin{array}{l}\text { - participação nas } \\
\text { decisões de interesse } \\
\text { geral }\end{array}$ \\
\hline $\begin{array}{l}\text { Subsistema de } \\
\text { operação/produção } \\
\text { de serviços }\end{array}$ & $\begin{array}{l}\text { - coerência com } \\
\text { planejamento central } \\
\text { (avaliação) } \\
\text { - integração com outras } \\
\text { partes do sistema }\end{array}$ & $\begin{array}{l}\text { - prestação de contas } \\
\text { (instâncias centrais/ } \\
\text { população) }\end{array}$ & $\begin{array}{l}\text { - criação de instâncias } \\
\text { representativas } \\
\text { - acesso à informação }\end{array}$ \\
\hline $\begin{array}{l}\text { Subsistema de } \\
\text { informação e } \\
\text { retroalimentação }\end{array}$ & $\begin{array}{l}\text { - critérios e indicadores } \\
\text { estratégicos } \\
\text { de acompanhamento } \\
\text { da gestão }\end{array}$ & $\begin{array}{l}\text { - critérios e } \\
\text { indicadores táticos } \\
\text { de acompanhamento } \\
\text { da gestão }\end{array}$ & $\begin{array}{l}\text { - acompanhamento } \\
\text { da gestão }\end{array}$ \\
\hline
\end{tabular}

Engl obando situações tão distintas, a categoria não pode ser unidimensional. Em termos processuais, só é possível alcançar todos esses objetivos em todas as suas dimensões e instâncias se o sistema de saúde estiver estrategicamente orientado por esse valor acesso, isto é, acesso das necessidades da população à pauta de valores e objetivos a serem levados em consideração pelas políticas dos diversos níveis do sistema.

A descentralização, por seu lado, não foi considerada um valor e um fim em si mesma, mas uma condição necessária para melhorar o acesso. Sua avaliação leva em consideração a missão e as funções do Estado, os serviços de saúde e os usuários do sistema. Com relação a essa categoria, o estudo desenvolvido até o momento sugere algumas questões que, no entanto, ainda ficam sem resposta. A primeira delas é sobre a possi bilidade de que a descentralização seja perfeita em uma dimensão (deci- são), mas tenha graus diferentes em outra (informação).

A segunda é sobre a possibilidade de justificar que haja uma descentralização perfeita política (autonomia local), mas que alguns serviços sejam menos descentralizados (oncologia) e que, mais ainda, a dimensão integração/informação seja imperfeita. A justificativa pode se calcar na afirmação de que a descentralização seja a melhor forma de se atingir o valor acesso. Não se defende al guma forma de descentralização como um valor per se, mas arranjos que garantiriam as dimensões de acesso.

A terceira questão busca associar as duas categorias em estudo: a acessibilidade geográfica é mais bem garantida por organizações política ou administrativamente descentralizadas, parcialmente integradas e sob regulação tecnocrática? Ainda não temos a resposta para essas perguntas, mas o trabalho empírico propiciará respondê-las. 


\section{Referências}

CONTANDRIOPOULOS, A. P., 1986. Analyse de la dynamique des systèmes de santé. Groupe de RechercheInterdisciplinaireen Santé, 86:31.

CONTANDRIOPOULOS, A. P., 1990. Recherche sur les fondements d'un modèle théorique du système de santé. Groupe de Recherche Interdisciplinaire en Santé, 90:11.

CONTANDRIOPOULOS, A. P., 1994. Réformer le système de santé: Une utopie pour sortir d'un statu quo impossible. Ruptures, Revue Transdisciplinaireen Santé, 1:8-26.

FOREST, P. G., s/d. La participation des citoyens à I'administration des services de santé. In: LeSystème de Santé au Quebéc: Organisations, Acteurs et Enjeux (V. Lenieux; P. Bergeron; C. Bégin \& G. Bélanger, org.), pp. 143-161, Sante Foy: Les Presses de l'Úniversité de Laval.

HEIDENHEIMER, A. J., 1986. L'étude comparative des politiques publiques: Une odysée en quatre étapes. Revue Internationale des Sciences Sociales, 108:171-192.
HORTALE, V. A.; CONILL, E. \& PEDROZA, M., 1999. Desafios na construção de um modelo para análise comparada da Organização de Serviços de Saúde. Cadernos de Saúde Pública, 15:79-88.

LE MOIGNE, J. L., 1990. La Modelisation des Systèmes Complexes. Paris: Dunod.

MÉNY, Y., 1993. PolitiqueComparée. Paris: Montchrestien.

ROEMER, M., 1991. National Systems in the World. New York: Oxford University Press.

RODRIGUE, J., 1990. Une Modélisation Systémique de L'épisode de Soins (memoire). Montréal: Université de Montréal.

SICOTTE, C.; CHAMPAGNE, F.; CONTANDRIOPOULOS, A. P.; BÉLAND, F.; DENIS, J. L.; BILODEAU, H.; BRÉMOND, M.; LEMIEUX-CHARLES, L.; BRASNLEY, J.; LEGGAT, S. \& BAKER, R., 1996. A Conceptual Framework for the Analysis of Health Care Organization Performance. Toronto: Health Services Management Research of Toronto. 